\title{
Hand Hygiene and Face Touching in Family Medicine Offices: A Cincinnati Area Research and Improvement Group (CARInG) Network Study
}

\author{
Nancy C. Elder, MD, MSPH, Will Sawyer, MD, Harini Pallerla, MS, Sara Khaja, MD, \\ and Monica Blacker, BS
}

Background: Family medicine offices may play an important role in the transmission of common illnesses such as upper respiratory tract infections (URTIs). There has, however, been little study of whether physicians teach patients about URTI transmission and what their own actions are to prevent infection. The purpose of this study was to assess the quality of hand hygiene and the frequency with which family physicians and staff touch their eyes, nose, and mouth (the T-zone) as well as physician and staff self-reported behaviors and recommendations given to patients regarding URTI prevention.

Methods: We observed family physicians and staff at 7 offices of the Cincinnati Area Research and Improvement Group (CARInG) practice-based research network for the quality of hand hygiene and number of T-zone touches. After observations, participants completed surveys about personal habits and recommendations given to patients to prevent URTIs.

Results: A total of $\mathbf{3 1}$ clinicians and 48 staff participated. They touched their T-zones a mean of 19 times in 2 hours (range, $0-105$ times); clinicians did so significantly less often than staff $(P<.001)$. We observed 123 episodes of hand washing and 288 uses of alcohol-based cleanser. Only 11 hand washings (9\%) met Centers for Disease Control and Prevention criteria for effective hand washing. Alcohol cleansers were used more appropriately, with 243 (84\%) meeting ideal use. Participants who were observed using better hand hygiene and who touched their T-zone less report the same personal habits and recommendations to patients as those with poorer URTI prevention hygiene.

Conclusions: Clinicians and staff in family medicine offices frequently touch their T-zone and demonstrate mixed quality of hand cleansing. Participants' self-rated URTI prevention behaviors were not associated with how well they actually perform hand hygiene and how often they touch their T-zone. The relationship between self-reported and observed behaviors and URTIs in family medicine office settings needs further study. ( $\mathrm{J}$ Am Board Fam Med 2014;27:339-346.)

Keywords: Infectious Diseases, Practice-based Research, Respiratory Tract Diseases

Family medicine offices have the potential to play an important role in the transmission of common infectious diseases such as upper respiratory tract infections (URTIs). These infections are responsi-

This article was externally peer reviewed.

Submitted 23 August 2013; revised 6 January 2014; accepted 13 January 2014.

From the Department of Family and Community Medicine, University of Cincinnati, Cincinnati, OH (MCE, HP); Sharonville Family Medicine, Cincinnati, Ohio (WS); the Bethesda Family Medicine Residency, Cincinnati, Ohio (SK); and the Northeast Ohio College of Medicine, Rootstown $(\mathrm{MB})$.

Funding: This project was supported by a Practice-Based Research Network Stimulation Grant from the American Academy of Family Physicians Foundation and by the ble for significant morbidity, lost work, and health care costs in the United States and are a common reason patients visit a family physician. Americans have 500 million noninfluenza viral URTIs every

National Center for Research Resources and the National Center for Advancing Translational Sciences, National Institutes of Health (grant no. 8 UL1 TR000077-04).

Conflict of interest: none declared.

Disclaimer: The content in this article is solely the responsibility of the authors and does not necessarily represent the official views of the National Institutes of Health or the American Academy of Family Physicians.

Corresponding author: Nancy C. Elder, MD, MSPH, Department of Family and Community Medicine, University of Cincinnati, PO Box 670566, Cincinnati, OH 45267-0566 (E-mail: nancy.elder@uc.edu). 
year, and $22 \%$ of these infections lead to an office visit. ${ }^{1}$ In addition to providing diagnosis and management during the family medicine visit, clinicians have an imperative to implement precautions to decrease spread, as well as an opportunity for patient education and role modeling to prevent future infections. $^{2}$

All recommendations to decrease the spread of URTIs include hand hygiene, that is, either washing hands with soap and water or using an alcohol-based cleanser. ${ }^{2-6}$ While several studies have documented ambulatory care clinicians' imperfect adherence to hand hygiene frequency recommendations, ${ }^{6-10}$ there are few studies that objectively document the quality of hand hygiene performed by primary care clinicians. ${ }^{11}$ The question remains: Are they cleaning their hands in a way that will decrease pathogens on their skin? Even the large amount of literature on hand hygiene in hospitals focuses primarily on adherence to performing hand hygiene, not the actual quality of the hand hygiene. ${ }^{12}$

Less well known is a common step in the transmission of many respiratory pathogens: self-inoculation from the hand to the mucus membranes of the eyes, nose, and mouth (the T-zone). ${ }^{13,14}$ While not all respiratory pathogens are transmitted via this route, many are, including the respiratory syncytial virus and rhinovirus, ${ }^{14}$ and anecdotal reports have found that decreased touching of the T-zone results in fewer URTIs. ${ }^{13,15}$ Touching the T-zone often occurs without thought, and studies have documented this in office workers (16 touches/ hour) ${ }^{15}$ and in grand round attendees, where 1 in 3 picked their nose and 1 in 2.7 rubbed their eyes. ${ }^{16}$

Family physicians and their staff serve as sources of patient education and role modeling. A recent study found that practicing a healthful behavior oneself was the most consistent predictor of physicians counseling patients about related prevention issues. ${ }^{17}$ Whether the practice of healthful behaviors to prevent URTIs, such as good hand hygiene and not touching the T-zone, is associated with other behaviors and patient education for URTI prevention is unknown, as are objective data about the quality of hand hygiene and T-zone touching in family medicine practice. Therefore, we observed family physicians and their staff at practices in the Cincinnati Area Research and Improvement Group (CARInG) practice-based research network $(\mathrm{PBRN})$ and assessed the quality of their hand hy- giene and the frequency with which they touched their own T-zone. In addition, we surveyed clinicians and staff about self-reported behaviors and recommendations given to patients regarding URTI prevention.

\section{Methods}

The CARInG Network is a regional primary care PBRN with 21 member practices. All practices were invited, and 7 chose to participate in this study. This study was approved by the University of Cincinnati Institutional Review Board.

\section{Participants}

Within each practice, each clinician (physician and nurse practitioner) and staff member who had contact with patients as part of their job (front office, medical assistants [MAs]), nurses, behavioral counselors, supervisors) could elect to participate in the study. To limit the likelihood that participants would change behaviors during the observation period, participants were told only that the study's purpose was to "describe family physicians' and staff's observed behaviors during their routine medical office work that might impact infection." Hand hygiene and touching the T-zone were never specifically mentioned to the participants.

\section{Data Collection}

\section{Observations}

Each participant was observed for a 2-hour period while performing their usual duties. During that time, the observer noted how many times the participant touched their eyes, nose, and/or mouth with their hands. In addition, each time the participant cleansed their hands, observers noted details about each component of an "ideal" cleansing., 5,12 For alcohol-based cleanser this entailed rubbing the hands together until the product covered all surfaces of the hands and waiting for product to dry completely. For hand washing, this entailed (1) wetting hands, (2) covering all surfaces of hands with soap/water, (3) rubbing the hands together for a minimum of 10 seconds, (4) drying hands with a disposable towel, and (5) using the towel to turn off the water or using an automatic faucet. Observers followed participants into the examination rooms with patients, but if the observers were asked not to follow a participant to an activity, the observer noted that pause and added additional time to the 
observation period. Three observers were trained by the study investigators (NCE, WS) and they practiced in a nonparticipating office until their observations of the key components described above were identical.

\section{Surveys}

After all observations were completed in an office, clinicians and staff completed a 2-page survey about personal behaviors and recommendations given to patients to prevent URTIs. Questions for the survey were taken from both the medical literature 2,3,6,7,18 as well as common activities in family medicine practice. There were 12 activities and recommendations presented to participants, 8 of which have been recommended by the Centers for Disease Control and Prevention (CDC) or are supported by the literature. ${ }^{3,19}$ A draft survey was pilot tested with staff and clinicians who were not involved in the study and was revised for clarity.

\section{Data Analysis}

The observation and the survey data were analyzed using SPSS software versions 19 and 20 (IBM, Chicago, IL). Touching the T-zone and quality of hand cleansing was determined for each participant, and we used $t$ tests and one-way analyses of variance to compare results by professional role and practice experience. We defined a subset of clinicians and staff who demonstrated less T-zone touching and better hand hygiene; these participants touched their T-zone less than the median number of touches for the entire group and rubbed their hands 10 seconds or more each time they washed with soap or used an alcohol-based cleanser correctly every time. $\chi^{2}$ Tests were used to assess differences in personal and recommended URTI prevention activities by observed behaviors. Because of the large number of individual survey items analyzed, ${ }^{20}$ we adjusted the $P$ value for significance to .002 using Bonferroni's correction.

\section{Results}

A total of 31 clinicians and 48 staff participated in the study. Participation by clinicians and staff at each site varied between $50 \%$ and $100 \%$; overall, $61 \%$ of the clinicians and $71 \%$ of the staff at these offices participated in the study. Participants' demographics are found in Table 1 . The 7 office sites included 2 community health centers, 1 family medicine residency, 2 offices affiliated with an academic health center, and 2 offices affiliated with a large health system—a breakdown representative of the entire CARInG PBRN.

\section{Observations}

Touching the T-zone

Participants touched their eyes, nose, and/or mouth a mean of 19 times in 2 hours (median, 15; range, $0-105)$. The mouth was touched twice as often as the nose or the eyes. In 2 hours, clinicians touched their T-zones significantly less than both nursing and other staff (clinicians, 10 touches; nursing staff, 18 touches; and other staff, 32 touches; $P<.001$ ). There were, however, no statistically significant differences be-

Table 1. Demographics of Observation and Survey Participants by Office Site

\begin{tabular}{|c|c|c|c|c|c|c|c|c|}
\hline Demographics & Total & Site 1 & Site 2 & Site 3 & Site 4 & Site 5 & Site 6 & Site 7 \\
\hline \multicolumn{9}{|l|}{ Staff, n } \\
\hline $\begin{array}{l}\text { Clinicians (physicians and } \\
\text { nurse practitioners) }\end{array}$ & 31 & 2 & 3 & 5 & 10 & 1 & 4 & 6 \\
\hline $\begin{array}{l}\text { Nursing staff (nurses and } \\
\text { medical assistants) }\end{array}$ & 26 & 1 & 4 & 5 & 4 & 1 & 2 & 9 \\
\hline Other & 22 & 0 & 3 & 4 & 3 & 4 & 3 & 5 \\
\hline Total & 79 & 3 & 10 & 14 & 17 & 6 & 9 & 20 \\
\hline Female sex $(\%)$ & 80 & 67 & 90 & 71 & 71 & 100 & 67 & 90 \\
\hline \multicolumn{9}{|l|}{ Race (\%) } \\
\hline White & 60 & 33 & 50 & 62 & 71 & 83 & 56 & 59 \\
\hline African American & 36 & 67 & 50 & 38 & 23 & 17 & 44 & 41 \\
\hline Other & 4 & & & & 6 & & & \\
\hline Mean age, years (range) & $40(23-82)$ & $42(41-44)$ & $41(25-62)$ & $44(32-60)$ & $38(25-60)$ & $55(32-82)$ & $34(23-52)$ & $36(25-57)$ \\
\hline $\begin{array}{l}\text { Mean years in profession } \\
\text { (range) }\end{array}$ & $12(0.6-42)$ & $13(3-20)$ & $12(2-40)$ & $11(1-30)$ & $9(0.6-40)$ & $26(6-42)$ & $12(1-27)$ & $9(0.5-30)$ \\
\hline
\end{tabular}


tween those participants who did and did not have direct contact with patients during the observation (17 vs 21 touches), those with different lengths of time in practice ( $<5$ years, 21 touches; $\geq 5$ years, 16 touches), or those who replied in the survey that they frequently avoided touching their eyes, nose, and mouth versus those who only occasionally or rarely avoided touching (19 vs 20 touches, respectively).

\section{Hand Hygiene}

We observed 123 episodes of hand washing by 39 individuals and 288 uses of alcohol-based cleanser by 58 individuals. Specifically, we observed a mean of 2 hand washes per clinician (range, $0-8$ hand washes), 2 per nursing/MA staff ( $0-7$ hand washes), and 1 per other staff ( $0-2$ hand washes). For the use of alcohol-based cleanser, we observed a mean of 6 uses per clinician (range, 0-7 uses), 2 per nursing/MA staff ( $0-12$ uses), and 1 per other staff ( $0-7$ uses).

\section{Ideal Hand Washing}

The components of an ideal hand washing that were usually done improperly were rubbing the hands with soap and water for an insufficient amount of time (mean, 8 seconds; range, 2-22 seconds) and not using a towel to turn off the faucet (or using an automatic faucet) (Figure 1). There were no statistical differences between clinicians and staff in the correct completion of these hand washing components. Only 11 of 123 hand washings (9\%) met all the ideal criteria, and these were performed by 3 clinicians and 2 nursing/MA staff. Therefore, only 13\% (5 of 39) of the clinicians and staff at these offices who washed their hands with soap and water during the observation periods did so correctly even once.

\section{Ideal Use of Alcohol-based Cleanser}

Participants used alcohol cleansers more appropriately, with 243 of 288 (84\%) meeting both criteria of ideal use. Incorrectly performed components were equally divided between not rubbing the cleanser over the entire hand and not allowing the hands to dry before touching something/someone. There were no significant differences between clinicians and staff in the quality of either form of hand cleansing.

\section{Survey}

Participants were asked whether they frequently, occasionally, or rarely performed any of a number of activities to prevent URTIs in themselves and whether they recommended the activity to patients. We specifically assessed whether observed behaviors were related to self-reported behaviors or recommendations to patients. We compared the percentage of self-reports of "frequently" making recommendations to patients or performing a behavior themselves between the group with "better" hand hygiene (fewer than the median number of T-zone touches and better hand hygiene) to the rest of the participants (Tables 2 and 3). Better hand hygiene was demonstrated by 26 participants

Figure 1. Percentage of soap-and-water hand-washing activities $(n=123)$ performed correctly by 39 clinicians, nurses, and other staff.

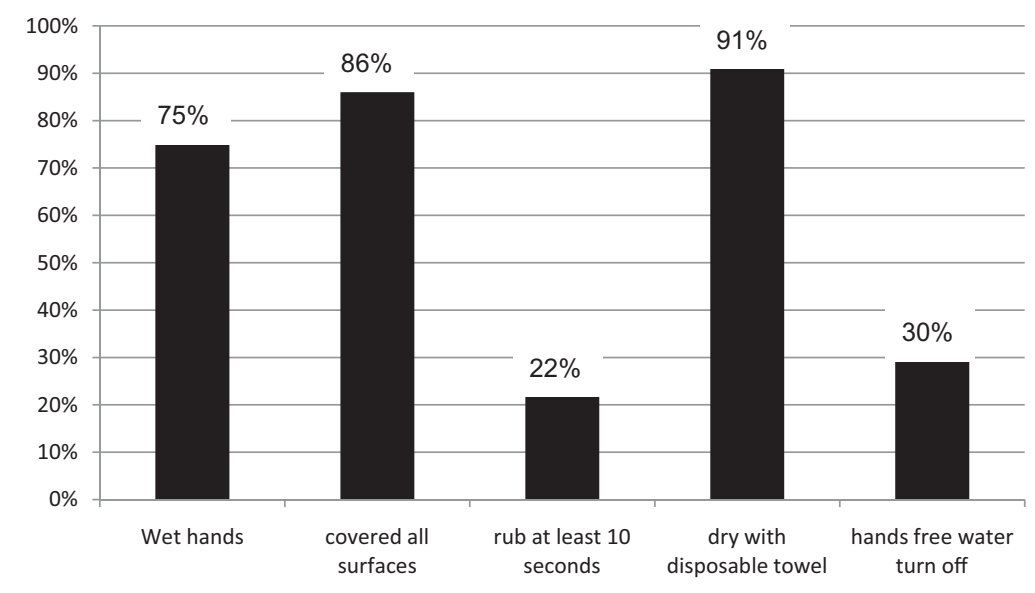

$\mathrm{N}=123$ hand washings (observed in 39 clinicians, nursing and other staff) 
Table 2. Percentages of Participants Who Report They Frequently Recommend Behaviors to Patients to Prevent Upper Respiratory Tract Infections (URTIs) by Level of Observed T-Zone Touching and Hand Hygiene Behaviors

\begin{tabular}{lcc}
\hline & $\begin{array}{c}\text { More Touching/Poor } \\
\text { Hygiene (n = 53) }\end{array}$ & $\begin{array}{c}\text { Less Touching/Better } \\
\text { Hygiene (n }=39)\end{array}$ \\
\hline Value
\end{tabular}

CDC, Centers for Disease Control and Prevention.

(15 clinicians, 6 MAs, and 5 other staff). There were no differences in the percentage of participants who stated they frequently performed or recommended any behavior by whether the participant was observed practicing better hand hygiene. While those observed with better hygine trended slightly away from non-recommended activities, there were no differences between the groups in the performance or recommendation to patients of CDC-recommended activities.

\section{Discussion}

Viral URTIs are common infections in family medicine. ${ }^{1}$ While many people are simply miserable for a few days, some develop serious complications that

Table 3. Percentages of Participants Who Report They Frequently Perform Behaviors to Prevent Upper Respiratory Tract Infections (URTIs) by Level of Observed T-Zone Touching and Hand Hygiene Behaviors

\section{More Touching/Poor Less Touching/Better}

Hygiene $(\mathrm{N}=53) \quad$ Hygiene $(\mathrm{N}=39) \quad P$ Value

Recommended by the CDC to prevent URTIs

Don't smoke or stop smoking if you smoke

Cover the mouth/nose with a tissue or handkerchief when coughing/

$68.1 \%$

54.7

sneezing

Cover the mouth/nose with the elbow when coughing/sneezing

Wash hands frequently throughout the day

Try to not touch own eyes, nose, or mouth with hands

Get plenty of sleep each night

Avoid being around people when they have a cold or flu

No recommendation by the CDC to prevent URTI

Take vitamin $\mathrm{C}$ regularly

Drink large amounts of water daily

Cover the mouth/nose with the hand when coughing/sneezing

Stay at home (do not go to school or work) while ill with a cold or flu

Avoid stressful situations

\begin{tabular}{lll}
92.5 & 88.5 & .56 \\
96.2 & 96.0 & .96 \\
47.2 & 46.2 & .93 \\
47.1 & 38.5 & .47 \\
33.3 & 23.1 & .35 \\
& & \\
25.0 & 11.5 & .16 \\
60.4 & 34.6 & .03 \\
52.9 & 50.0 & .81 \\
20.0 & 15.4 & .62 \\
26.0 & 20.0 & .57 \\
\hline
\end{tabular}

CDC, Centers for Disease Control and Prevention. 
lead to hospitalizations and death. Prevention of URTIs is a key component of public health campaigns by the CDC and state and local health departments. ${ }^{3}$ Nevertheless, there are few objective studies of the quality of hand hygiene and no studies of T-zone touching in primary care. We found that family medicine clinicians and staff often touch their T-zones and inconsistently practice good hand hygiene; we also noted that their observed behaviors were unrelated to self-reported behaviors for URTI prevention.

Hand washing is a simple task, learned during childhood, yet its use by health care workers is complex. ${ }^{11,21}$ Reported barriers to hand hygiene include being too busy; lack of easy access to sinks, soap, and paper towels; and simply forgetting. ${ }^{5}$ The few studies of appropriate hand-washing frequency in the outpatient setting have documented appropriateness between $31 \%$ (in dermatology) to $52 \%$ (in family medicine.) ${ }^{8-10}$ Details about the quality of the hand washing are lacking. ${ }^{11}$ While some authors have noted that washing hands with soap and water would take at least 60 seconds to be performed properly, ${ }^{12}$ others have found that contact times as short as 10 seconds are effective in reducing transient hand flora. ${ }^{22}$ In our study, only $22 \%$ of the observed hand washings met this conservative recommendation of 10 seconds. Alcoholbased hand cleansers are quicker and simpler to use and were usually used correctly in these family medicine offices. While not suitable in all situations (such as when hands are visibly soiled), alcoholbased cleansers seem an appropriate agent of choice for hand hygiene in primary care and an effective method of decreasing URTIs; their availability and use in colleges and military facilities documented significantly lower rates of URTIs. ${ }^{23,24}$

To our knowledge, this is the first study documenting the frequency of touching the T-zone among health care workers during the performance of their duties. CDC guidelines include "avoid touching your eyes, nose, and mouth" among other recommendations to prevent the flu. ${ }^{3}$ However, touching the T-zone has not received the same study as hand hygiene. Clinical trials documenting improvement in rates of respiratory infection by eliminating this behavior are lacking, and we must depend on anecdotal reports ${ }^{13,15}$ and laboratory studies of self-inoculation ${ }^{14,16}$ to justify this recommendation. Because studies of touching the T-zone are so limited, it is unknown whether the rates seen by these family medicine clinicians and staff are more or less than other professionals. The rate exhibited by staff in this study (32 touches/2 hours) is similar to that reported among office workers (16 touches/hour), ${ }^{15}$ which would be expected since nonclinical staff often answer phones and work on computers, much like office workers. Clinicians touched their T-zones less than MA/nursing staff, but all groups were similar in the quality of their hand washing. It may be that more clinicians were aware of URTI transmission via the T-zone than MAs/nurses, but further study is needed to confirm this possibility. Touching the face is a habitual behavior for most people and as such often goes unnoticed. This could account for our finding that those participants who stated they frequently avoided touching their face actually touched it at the same rate as those who reported only occasionally or rarely touching their face.

Clinicians and staff who use proper hand hygiene and avoid touching the T-zone serve as role models for patients and for each other. Even without spoken encouragement, the mere act of watching a colleague or a trusted professional perform a behavior makes it more likely that an individual will engage in that behavior. This "nonconscious behavioral mimicry" smoothes interpersonal interactions and serves as social glue. ${ }^{20,25}$ While successful infection prevention programs in medical settings have included behavior modeling by opinion leaders and champions, ${ }^{7,18,21,26}$ it was never used as a sole method of prevention. This is consistent with our finding of no association between observed better hand hygiene/limited T-zone touching and self-reported behaviors for either personal protection against URTIs or provision of URTI prevention education to patients. Changing URTI prevention attitudes and behaviors, and hopefully clinical outcomes, will take culture, process, and system changes, not just role modeling.

There are limitations to this study. It took place in only one geographic area, and behaviors in other areas might differ. While we did not inform participants that we were observing the quality of hand hygiene and touches to the T-zone, ethically we were required to tell participants that our study was related to infection prevention practices. This, and the fact participants were being observed, may have changed the actual behaviors practiced by participants during the observation period. However, hand hygiene was still performed poorly by most 
participants and the T-zone was frequently touched. We were unable to assess whether hand hygiene occurred at appropriate times, but, as noted previously, there is existing research on this in outpatient medicine $^{8-10}$; our goal was to assess the quality of hand hygiene, a rarely study component.

\section{Conclusions}

URTIs are responsible for significant morbidity and are a common reason patients visit a family physician. During office visits, clinicians can implement precautions to protect themselves, decrease spread, provide patient education, and role model preventive behaviors such as good hand cleansing and not touching the T-zone. ${ }^{2}$ While the literature has shown that ambulatory physicians and nurses often miss appropriate opportunities to perform hand hygiene, this is the first study to document how often primary care clinicians and staff touch the T-zone and is one of few to document how well hand cleansing is performed. We found that family medicine clinicians and staff frequently touch their T-zone and that the quality of hand hygiene is relatively good for alcohol-based cleanser but poor for soap and water. Those who were observed practicing better hand hygiene were no more likely to self-report frequent use of personal URTI prevention habits nor recommend good URTI prevention strategies to their patients. Further research is needed to assess whether these findings are typical of family medicine offices and to determine whether changing hand hygiene or T-zone touching behaviors would have an effect on URTI prevention strategies or patient outcomes.

\section{References}

1. Fendrick AM, Monto AS, Nightengale B, Sarnes M. The economic burden of non-influenza-related viral respiratory tract infection in the United States. Arch Intern Med 2003;163:487-94.

2. Hogg W, Huston P. Controlling droplet-transmitted respiratory infections: best practices and cost. Can Fam Physician 2006;52:1229-32.

3. Everyday preventive actions that can help fight germs, like flu. Atlanta: Centers for Disease Control and Prevention, National Center for Immunization and Respiratory Diseases; 2010. Available from: http://www.cdc.gov/flu/pdf/freeresources/updated/ everyday_preventive.pdf. Accessed July 7, 2013.

4. Prevention and control of healthcare-associated infections in primary and community care. London:
National Institute for Health and Clinical Excellence; 2012.

5. Boyce JM, Pittlet D; Healthcare Infection Control Practices Advisory Committee; HICPAC/SHEA/ APIC/IDSA Hand Hygiene Task Force. Guideline for hand hygiene in health-care settings. Recommendations of the Healthcare Infection Control Practices Advisory Committee and the HICPAC/ SHEA/APIC/IDSA Hand Hygiene Task Force. Society for Healthcare Epidemiology of America/ Association for Professionals in Infection Control/ Infectious Diseases Society of America. MMWR Recomm Rep. 2002;51(RR-16):1-45.

6. Turnberg $W$, Daniell $W$, Seixas $N$, et al. Appraisal of recommended respiratory infection control practices in primary care and emergency department settings. Am J Infect Control 2008;36:268-75.

7. Hogg W, Huston P, Martin C, et al. Promoting best practices for control of respiratory infections: collaboration between primary care and public health services. Can Fam Physician 2006;52:1110-1.

8. Cohen H, Kitai E, Levy I, Ben-Amitai D. Handwashing patterns in two dermatology clinics. Dermatology 2002;205:358-61.

9. Cohen HA, Matalon A, Amir J, Paret G, Barzilai A. Handwashing patterns in primary pediatric community clinics. Infection 1998;26:45-7.

10. Whyte B, Corea M, Brill J, Baumgardner D. Handwashing frequency and factors that influence it in a family practice clinic. J Am Board Fam Med 1997; 10:229-31.

11. Smith SM. A review of hand-washing techniques in primary care and community settings. J Clin Nurs 2009;18:786-90.

12. Marra AR, Edmond MB. Hand hygiene: state-ofthe-art review with emphasis on new technologies and mechanisms of surveillance. Curr Infect Dis Rep 2012;14:585-91.

13. Bertsch R. Avoiding upper respiratory tract infections by not touching the face. Arch Intern Med 2010;170:833-4.

14. Goldmann DA. Transmission of viral respiratory infections in the home. Pediatr Infect Dis J 2000; 19(10 Suppl):S97-102.

15. Nicas M, Best D. A study quantifying the hand-toface contact rate and its potential application to predicting respiratory tract infection. J Occup Environ Hyg 2008;5:347-52.

16. Hendley JO, Wenzel RP, Gwaltney JM Jr. Transmission of rhinovirus colds by self-inoculation. N Engl J Med 1973;288:1361-4.

17. Frank E. Physician health and patient care. JAMA 2004;291:637.

18. Huston P, Hogg W, Martin C, Soto E, Newbury A. A process evaluation of an intervention to improve respiratory infection control practices in family physician offices. Can J Public Health 2006;97:475-9. 
19. Cohen S, Doyle WJ, Alper CM, Janicki-Deverts D, Turner RB. Sleep habits and susceptibility to the common cold. Arch Intern Med 2009;169:62-7.

20. Chartrand T, Bargh J. The chameleon effect: the perception-behavior link and social interaction. J Personal Social Psychol 1999;76:893-910.

21. Pittet D, Simon A, Hugonnet S, Pessoa-Silva CL, Sauvan V, Perneger TV. Hand hygiene among physicians: performance, beliefs, and perceptions. Ann Intern Med 2004;141:1-8.

22. Sickbert-Bennett EE, Weber DJ, Gergen-Teague MF, Sobsey MD, Samsa GP, Rutala WA. Comparative efficacy of hand hygiene agents in the reduction of bacteria and viruses. Am J Infect Control 2005;33:67-77.
23. Ryan MA, Christian RS, Wohlrabe J. Handwashing and respiratory illness among young adults in military training. Am J Prev Med 2001;21:79-83.

24. White C, Kolble R, Carlson R, et al. The effect of hand hygiene on illness rate among students in university residence halls. Am J Infect Control 2003;31: 364-70.

25. Cheng C, Chartrand T. Self-monitoring without awareness: using mimicry as a nonsconscious affiliation strategy. J Personal Social Psychol 2003;85: 1170-9.

26. Lewis C, Clancy C, Leake B, Schwartz J. The counseling practices of internists. Ann Intern Med 1991; 114:46-53. 